\title{
Limiting Profile of the Blow-up Solutions for the Fourth-order Nonlinear Schrödinger Equation
}

\author{
Shihui Zhu, Jian Zhang, and Han Yang \\ Communicated by Y. Charles Li, received March 1, 2010.
}

\begin{abstract}
This paper is concerned with the blow-up solutions of the focusing fourth-order mass-critical nonlinear Schrödinger equation. Establishing the profile decomposition of the bounded sequences in $H^{2}$, we obtain the variational characteristics of the corresponding ground state and a compactness lemma. Moreover, we obtain the $L^{2}$-concentration of the blow-up solutions and the limiting profile of the minimal mass blow-up solutions in the general case.
\end{abstract}

\section{Contents}

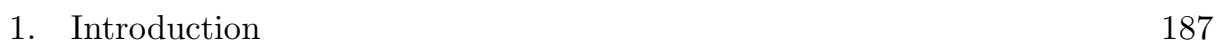

2. Preliminary 191

3. Variational Structure 195

4. $L^{2}$-Concentration 200

5. Limiting Profile of Minimal Mass Blow-up Solutions 201

\section{Introduction}

In this paper, we study the Cauchy problem of the focusing fourth-order masscritical nonlinear Schrödinger equation

$$
\begin{gathered}
i u_{t}-\triangle^{2} u+|u|^{\frac{8}{N}} u=0, t \in[0, T), x \in R^{N}, \\
u(0, x)=u_{0},
\end{gathered}
$$

2000 Mathematics Subject Classification. Primary 35B44, 35Q55.

Key words and phrases. Nonlinear Schrödinger equation, Blow-up solution, Profile decomposition, Limiting profile.

Supported by National Natural Science Foundation of P.R.China (10771151) and Fundamental Research Funds for the Central Universities (SWJTU09ZX073 and SWJTU09ZT36). 
where $i=\sqrt{-1} ; \triangle^{2}=\triangle \triangle$ is the biharmonic operator defined in $R^{N}$ and $\triangle=$ $\sum_{j=1}^{N} \frac{\partial^{2}}{\partial x_{j}^{2}}$ is the Laplace operator in $R^{N} ; u=u(t, x):[0, T) \times R^{N} \rightarrow \mathcal{C}$ is the complex valued function and $0<T \leq+\infty ; N$ is the space dimension. Fourthorder Schrödinger equations are introduced by Karpman [1] and Karpman, Shagalov $\mathbf{1 2}$ to take into account the role of small fourth-order dispersion terms in the propagation of intense laser beams in a bulk medium with Kerr nonlinearity, and such fourth-order Schrödinger equation are written as

$$
i \phi_{t}+\varepsilon \triangle^{2} \phi+\mu \triangle \phi+|\phi|^{p-1} \phi=0, \quad \phi=\phi(t, x): R \times R^{N} \rightarrow \mathcal{C} .
$$

Note that Equation (1.1) is a special case of Equation (1.3) by taking $\varepsilon=1$, $\mu=0$ and $p=1+\frac{8}{N}$. For the Cauchy problem (1.1)-(1.2), Kenig, Ponce and Vega 13, Ben-Artzi, Koch and Saut $[2$ established the local well-posedness in $H^{2}=H^{2}\left(R^{N}\right)$. Equation (1.1) is called the mass-critical due to the mass $M(u)=$ $\int_{R^{N}}|u(t, x)|^{2} d x$ and the equation itself are invariant under the rescaling symmetry $u \mapsto \lambda^{\frac{N}{2}} u\left(\lambda^{4} t, \lambda x\right)$.

We recall some known results for the classical focusing mass-critical nonlinear Schrödinger equation

$$
i v_{t}+\triangle v+|v|^{\frac{4}{N}} v=0, \quad v(0, x)=\varphi \in H^{1} .
$$

Ginibre and Velo [8] showed the local well-posedness in $H^{1}=H^{1}\left(R^{N}\right)$. In fact, in this space energy arguments apply, and a blow-up theory has been developed in the last two decades (see 4, 29] and the references therein). This theory is connected to the notion of ground state: the unique positive radial solution of the elliptic problem

$$
\triangle R-R+|R|^{\frac{4}{N}} R=0, \quad R \in H^{1}
$$

Weinstein 33 exhibited the following refined Gagliardo-Nirenberg inequality:

$$
\|f\|_{L^{2+\frac{4}{N}}}^{2+\frac{4}{N}} \leq\left(1+\frac{2}{N}\right)\left(\frac{\|f\|_{L^{2}}}{\|R\|_{L^{2}}}\right)^{\frac{4}{N}}\|\nabla f\|_{L^{2}}^{2}, \quad f \in H^{1} .
$$

Combined with the conservation of energy, this implies that: if the initial data $\|\varphi\|_{L^{2}}<\|R\|_{L^{2}}$, then the solution $v(t, x)$ exists globally; if the initial data $\|\varphi\|_{L^{2}} \geq$ $\|R\|_{L^{2}}$, then the solution $v(t, x)$ may blow up. The value $\|R\|_{L^{2}}$ is the sharp value of blow-up and global existence of the solutions in terms of Merle's [17 results. Using the variational characteristic of the ground state elliptic equation (1.5), Weinstein 34 showed the structure and formation of singularity of the minimal-mass blow-up solution (i.e. $\|\varphi\|_{L^{2}}=\|R\|_{L^{2}}$ ). It reads that the corresponding blow-up solution $v(t, x)$ remains close to $R(x)$ in $H^{1}$ up to scaling and phase parameters, and also translation in the nonradial case. In other words, the blow-up solution has the same shape as the ground state $R(x)$. Thus, basing on this structure and formation of singularity, Merle and Raphaël [18, 19] obtained a large body of breakthrough work on the qualitative properties of blow-up solutions with the help of the Spectral Properties [18, such as blow-up rates, profiles of blow-up solutions, etc. On the other hand, for $\varphi \in H^{1}$, Merle and Tsutsumi 20 30 (for radial data), Nawa 23 and Weinstein 35 (for general data) proved the following $L^{2}$-concentration property of the blow-up solutions by using the variational characterization of the ground state: 
there exists $x(t) \in R^{N}$ such that $\forall r>0$

$$
\lim _{t \rightarrow \tau} \inf \int_{|x-x(t)| \leq r}|v(t, x)|^{2} d x \geq \int R^{2} d x,
$$

where $\tau$ is the blow-up time. These results are extended to $\varphi \in H^{s}\left(R^{N}\right)$ for some $s_{0}<s<1$ (see $3, \mathbf{5}, \mathbf{1 0}, \mathbf{1 5}, \mathbf{3 1}, 32$ ) by using the variational characteristic of the ground state elliptic Equation (1.5) and harmonic analysis techniques.

In Equation (1.1), if we replace the nonlinearity $|u|^{\frac{8}{N}} u$ with $|u|^{p-1} u$, it is a class of semilinear fourth-order Schrödinger equations similar to Equation (1.1), which has been widely investigated. For $1<p<\frac{2 N}{(N-4)^{+}}$(where $\frac{2 N}{(N-4)^{+}}=+\infty$, when $N \leq 4 ; \frac{2 N}{(N-4)^{+}}=\frac{2 N}{N-4}$, when $N>4$ ), Ben-Artzi, Koch and Saut 2] established the local well-posedness in $H^{2}$. Fibich, Ilan and Papanicolaou $\mathbf{6}$ obtain the general results of global well-posedness in $H^{2}$. Pausader [25] and Segata 28] studied the global well-posedness and scattering of the fourth-order nonlinear Schrödinger equation with cubic nonlinearity. For $p=\frac{2 N}{N-4}$, Miao, Xu and Zhao [21, Pausader [26] studied the global existence and scattering of the focusing fourth-order nonlinear Schrödinger equation; Miao, Xu and Zhao [22, Pausader 24] studied the global existence and scattering of the defocusing fourth-order nonlinear Schrödinger equation. The above studies focused on global solutions. From the view-point of physics, physicists are very interested in the elaborate description of the blow-up solutions in $H^{2}$, such as blow-up rate, $L^{2}$-concentration, limiting profile of the blow-up solutions, etc.

In this paper, we study the limiting profile of the blow-up solutions to the Cauchy problem (1.1)-(1.2) in $H^{2}$. Motivated by the study of the classical masscritical nonlinear Schrödinger equation (1.4), we consider the ground state solution of the Equation (1.1)

$$
\triangle^{2} Q+Q-|Q|^{\frac{8}{N}} Q=0, \quad Q \in H^{2},
$$

which is a special periodic solution of Equation (1.1) in the form $u(t, x)=Q(x) e^{i t}$.

Fibich, Ilan and Papanicolaou [6] showed some numerical observations of the solution to the Cauchy problem (1.1)-(1.2), which implies that if the initial data $\left\|u_{0}\right\|_{L^{2}}<\|Q\|_{L^{2}}$, then the solution $u(t, x)$ exists globally; if the initial data $\left\|u_{0}\right\|_{L^{2}} \geq$ $\|Q\|_{L^{2}}$, then the solution $u(t, x)$ may blow up in finite time. Since the effect of fourth-order dispersion $\triangle^{2} u$, whether the variance identity arguments can be extended to show the existence of blow-up solutions for the biharmonic nonlinear Schrödinger equation is still unknown (see 1,6 ). On the other hand, the numerical observations in $\mathbf{6}$ showed the existence of blow-up solutions. Baruch, Fibich and Mandelbaum 1 obtained some dynamical properties of the radially symmetric blow-up solutions, such as blow-up rate, $L^{2}$-concentration. However, to our knowledge, the existence of ground state of the elliptic Equation (1.7), the variational structure of the ground state solution $Q$ and the limiting profile of the nonradially symmetric blow-up solutions are not addressed.

In the present paper, establishing the profile decomposition of the bounded sequences in $H^{2}$, we prove the existence of the ground state of elliptic Equation (1.7), and we obtain the variational characteristics of the ground state solution $Q(x)$. Moreover, we obtain a compactness lemma adapted to the analysis of the blow-up phenomenon of the fourth-order nonlinear Schrödinger equations in $H^{2}$, as follows. 
Theorem 1.1. Let $\left\{v_{n}\right\}_{n=1}^{\infty}$ be a bounded family of $H^{2}$ functions such that

$$
\lim _{n \rightarrow \infty} \sup \left\|\triangle v_{n}\right\|_{L^{2}} \leq M \quad \text { and } \quad \lim _{n \rightarrow \infty} \sup \left\|v_{n}\right\|_{L^{2+\frac{8}{N}}} \geq m .
$$

Then, there exists a sequence $\left\{x_{n}\right\}_{n=1}^{\infty}$ of $R^{N}$ such that up to a subsequence

$$
v_{n}\left(x+x_{n}\right) \rightarrow V(x) \text { weakly in } H^{2} .
$$

with $\|V\|_{L^{2}}^{\frac{8}{N}} \geq \frac{\|Q\|_{L^{2}}^{\frac{8}{N}} m^{2+\frac{8}{N}}}{\left(1+\frac{4}{N}\right) M^{2}}$, and $Q$ is the solution of ground state Equation [1.7).

Finally, we apply them to obtain some dynamical properties of the blow-up solutions in the general case: the $L^{2}$-concentration of the blow-up solutions, limiting profile of the minimal-mass blow-up solutions, as follows.

Theorem 1.2. Let $u(t, x) \in C\left([0, T) ; H^{2}\right)$ be the corresponding blow-up solution of the Cauchy problem (1.1)-(1.2). Suppose that $a(t)>0$ is any function such that

$$
\lim _{t \rightarrow T} a(t)=0 \text { and } \lim _{t \rightarrow T} \frac{(T-t)^{\frac{1}{4}}}{a(t)}=0 .
$$

Then, there exists $y(t) \in R^{N}$ such that

$$
\lim _{t \rightarrow T} \inf \int_{|x-y(t)| \leq a(t)}|u(t, x)|^{2} d x \geq \int|Q|^{2} d x,
$$

where $Q$ is the ground state solution of Equation (1.7).

Theorem 1.3. Let $u_{0} \in H^{2}$ and $\left\|u_{0}\right\|_{L^{2}}=\|Q\|_{L^{2}}$. Suppose that $u(t, x) \in$ $C\left([0, T) ; H^{2}\right)$ is the blow-up solution of the Cauchy problem (1.1)- 1.2) in finite time $0<T<+\infty$. Then $\forall \varepsilon>0, \exists \delta>0$ s.t. when $|t-T|<\delta$, there are functions $y(t) \in R^{N}, \gamma(t) \in R$ such that

$$
\left\|\lambda^{\frac{N}{2}}(t) u(t, \lambda(t)(x+y(t))) e^{i \gamma(t)}-Q(x)\right\|_{H^{2}}<\varepsilon,
$$

where $\lambda(t)=\left(\frac{\|\triangle Q\|_{L^{2}}}{\|\triangle u\|_{L^{2}}}\right)^{\frac{1}{2}}$ and $Q(x)$ is the unique solution of ground state Equation 1.7).

In this paper, the main tools of the proof of the compactness lemma in this paper are an argument of profile decomposition, introduced by Gérard $\mathbf{7}$ and Hmidi and Keraani 9 to study the defect of compactness for Sobolev embedding. We obtain the existence of ground state of the elliptic Equation (1.7) and variational characteristic of the ground state $Q(x)$ of Equation (1.7) by establishing the profile decomposition of the bounded sequence in $H^{2}$, which which are important in studying the blow-up dynamic of the blow-up solutions for fourth-order nonlinear Schrödinger equations. Moreover, we extend the results in [1] to the nonradially blow-up solutions of the Cauchy problem (1.1)-(1.2).

In this paper, We use the denotes $L^{q}:=L^{q}\left(R^{N}\right),\|\cdot\|_{q}:=\|\cdot\|_{L^{q}\left(R^{N}\right)}, H^{s}:=$ $H^{s}\left(R^{N}\right), \dot{H}^{s}:=\dot{H}^{s}\left(R^{N}\right)$ and $\int \cdot d x:=\int_{R^{N}} \cdot d x$. The various positive constants will be simply denoted by $C$. 


\section{Preliminary}

For the Cauchy problem (1.1)-(1.2), the energy space $H^{2}$ is defined by

$$
H^{2}:=\left\{\left.u \in S\left(R^{N}\right)\left|\int_{R^{N}}\left(1+|\xi|^{2}\right)^{2}\right| \widehat{u}(\xi)\right|^{2} d \xi<+\infty\right\}
$$

with the norm

$$
\|u\|_{H^{2}}=\left(\|u\|_{2}^{2}+\|\nabla u\|_{2}^{2}+\|\Delta u\|_{2}^{2}\right)^{\frac{1}{2}} .
$$

$H^{2}$ is a Hilbert space. It is easy to check that, there exist two positive constants $C_{1}>0$ and $C_{2}>0$ such that

$$
C_{1}\left(\|u\|_{2}^{2}+\|\triangle u\|_{2}^{2}\right) \leq\|u\|_{H^{2}}^{2} \leq C_{2}\left(\|u\|_{2}^{2}+\|\triangle u\|_{2}^{2}\right)
$$

which implies that $\left(\|u\|_{2}^{2}+\|\triangle u\|_{2}^{2}\right)^{\frac{1}{2}}$ is an equivalent norm of $H^{2}$. In this paper, we shall use this equivalent norm of $H^{2}:\left(\|u\|_{2}^{2}+\|\Delta u\|_{2}^{2}\right)^{\frac{1}{2}}$ to study the profile decomposition of the bounded sequences in $H^{2}$. Moreover, we define the energy functional $E(u)$ on $H^{2}$ by

$$
E(u):=\frac{1}{2} \int|\triangle u|^{2} d x-\frac{1}{2+\frac{8}{N}} \int|u|^{2+\frac{8}{N}} d x .
$$

The functional $E(u)$ is well-defined according to the Sobolev embedding theorem(see [4]). Ben-Artzi, Koch and Saut [2] established the local well-posedness of the Cauchy problem (1.1)-(1.2) in $H^{2}$, as follows.

Proposition 2.1. Let $u_{0} \in H^{2}$. There exists an unique solution $u(t, x)$ of the Cauchy problem (1.1)-(1.2) on the maximal time interval $[0, T)$ such that $u(t, x) \in$ $C\left([0, T) ; H^{2}\right)$ and either $T=+\infty$ (global existence), or else $0<T<+\infty$ and $\lim _{t \rightarrow T}\|u(t, x)\|_{H^{2}}=+\infty$ (blow-up). Furthermore, for all $t \in[0, T), u(t, x)$ satisfies the following conservation laws:

(i) Conservation of mass

(ii) Conservation of energy

$$
\int|u(t, x)|^{2} d x=\int\left|u_{0}\right|^{2} d x
$$

$$
E(u(t, x))=E\left(u_{0}\right) .
$$

Baruch, Fibich and Mandelbaum 1] obtained the lower-bound for the blow-up rate of the blow-up solutions to the Cauchy problem (1.1)-(1.2).

Lemma 2.2. Let $u(t, x)$ be the blow-up solution of the Cauchy problem (1.1)1.2) at finite time $0<T<+\infty$. Then, there exists a constant $K=K\left(\left\|u_{0}\right\|_{2}\right)>0$ such that

$$
\|\triangle u(t)\|_{2} \geq \frac{K}{(T-t)^{\frac{1}{2}}}, \quad 0<t<T .
$$

In order to study the variational characteristic of the ground state corresponding to Equation (1.1), we need the following profile decomposition of the bounded sequences in $H^{2}$, which is also our main tool. Similar results for bounded sequences in $L^{2}$ and $H^{1}$ have appeared in Gérard [7] and Hmidi and Keraani [9].

Proposition 2.3. Let $\left\{v_{n}\right\}_{n=1}^{\infty}$ be a bounded sequence in $H^{2}$. Then there exist a subsequence of $\left\{v_{n}\right\}_{n=1}^{\infty}$ (still denoted $\left\{v_{n}\right\}_{n=1}^{\infty}$ ), a family $\left\{x_{n}^{j}\right\}_{j=1}^{\infty}$ of sequences in $R^{N}$ and a sequence $\left\{V^{j}\right\}_{j=1}^{\infty}$ of $H^{2}$ functions such that 
(i) for every $k \neq j$,

$$
\left|x_{n}^{k}-x_{n}^{j}\right| \rightarrow \infty \text { as } n \rightarrow \infty,
$$

(ii) for every $l \geq 1$ and every $x \in R^{N}$

$$
v_{n}(x)=\sum_{j=1}^{l} V^{j}\left(x-x_{n}^{j}\right)+v_{n}^{l}(x),
$$

with

$$
\begin{aligned}
& \qquad \lim _{n \rightarrow \infty} \sup \left\|v_{n}^{l}\right\|_{p} \rightarrow 0, \text { as } l \rightarrow \infty, \\
& \text { for every } p \in\left(2, \frac{2 N}{(N-4)^{+}}\right) .
\end{aligned}
$$

Moreover, as $n \rightarrow \infty$, we have

$$
\left\|v_{n}\right\|_{2}^{2}=\sum_{j=1}^{l}\left\|V^{j}\right\|_{2}^{2}+\left\|v_{n}^{l}\right\|_{2}^{2}+o(1)
$$

and

$$
\left\|\triangle v_{n}\right\|_{2}^{2}=\sum_{j=1}^{l}\left\|\triangle V^{j}\right\|_{2}^{2}+\left\|\triangle v_{n}^{l}\right\|_{2}^{2}+o(1) .
$$

Proof. Since $H^{2}$ is a Hilbert space, we denote $\mu\left(v_{n}\right)$ is the set of functions obtained as weak limits of subsequences of the translated $v_{n}\left(x+x_{n}\right)$ with $\left\{x_{n}\right\}_{n=1}^{\infty}$ in $H^{2}$. We denote

$$
\eta\left(v_{n}\right)=\sup \left\{\|V\|_{2}+\|\triangle V\|_{2}, V \in \mu\left(v_{n}\right)\right\} .
$$

It is obvious that

$$
\eta\left(v_{n}\right) \leq \lim _{n \rightarrow \infty} \sup \left\{\|V\|_{2}+\|\triangle V\|_{2}\right\} .
$$

Next, we shall prove that there exist a subsequence $\left\{V^{j}\right\}_{j=1}^{\infty}$ of $\mu\left(v_{n}\right)$ and a family $\left\{x_{n}^{j}\right\}_{j=1}^{\infty}$ of sequences of $R^{N}$ such that

$$
\forall k \neq j \quad\left|x_{n}^{k}-x_{n}^{j}\right| \rightarrow \infty \text { as } n \rightarrow \infty
$$

and up to extracting a subsequence, the sequence $\left\{v_{n}\right\}_{n=1}^{\infty}$ can be written as

$$
v_{n}(x)=\sum_{j=1}^{l} V^{j}\left(x-x_{n}^{j}\right)+v_{n}^{l}, \eta\left(v_{n}^{l}\right) \rightarrow 0(l \rightarrow \infty)
$$

and (2.7) and (2.8) are true.

Indeed, if $\eta\left(v_{n}\right)=0$, we can take $V^{j}=0$ for all $j$, otherwise, we choose $V^{1} \in \mu\left(v_{n}\right)$ such that

$$
\left\|\triangle V^{1}\right\|_{2}+\left\|V^{1}\right\|_{2} \geq \frac{1}{2} \eta\left(v_{n}\right)>0 .
$$

By the definition of $\mu\left(v_{n}\right)$, there exists a subsequence $x_{n}^{1}$ of $R^{N}$ such that up to extracting a subsequence, we have

$$
v_{n}\left(x+x_{n}^{1}\right) \rightarrow V^{1}(x) \text { weakly in } H^{2} .
$$

Setting $v_{n}^{1}(x)=v_{n}(x)-V^{1}\left(x-x_{n}^{1}\right)$, by (2.13), we have $v_{n}^{1}\left(x+x_{n}^{1}\right) \rightarrow 0$ weakly in $H^{2}$ and

$$
\left\|v_{n}\right\|_{2}^{2}=\left\|V^{1}\right\|_{2}^{2}+\left\|v_{n}^{1}\right\|_{2}^{2}+o(1)
$$




$$
\left\|\triangle v_{n}\right\|_{2}^{2}=\left\|\triangle V^{1}\right\|_{2}^{2}+\left\|\triangle v_{n}^{1}\right\|_{2}^{2}+o(1) .
$$

Now, replacing $v_{n}$ by $v_{n}^{1}$ and repeating the same process. There exists $V^{2} \in \mu\left(v_{n}\right)$ such that $\left\|\triangle V^{2}\right\|_{2}+\left\|V^{2}\right\|_{2} \geq \frac{1}{2} \eta\left(v_{n}^{1}\right)>0$ and

$$
v_{n}^{1}\left(x+x_{n}^{2}\right) \rightarrow V^{2}(x) \text { weakly in } H^{2} .
$$

Setting $v_{n}^{2}(x)=v_{n}^{1}(x)-V^{2}\left(x-x_{n}^{2}\right)$, by (2.13), we have $v_{n}^{2}\left(x+x_{n}^{2}\right) \rightarrow 0$ weakly in $H^{2}$ and

$$
\begin{gathered}
\left\|v_{n}^{1}\right\|_{2}^{2}=\left\|V^{2}\right\|_{2}^{2}+\left\|v_{n}^{2}\right\|_{2}^{2}+o(1), \\
\left\|\triangle v_{n}^{1}\right\|_{2}^{2}=\left\|\triangle V^{2}\right\|_{2}^{2}+\left\|\triangle v_{n}^{2}\right\|_{2}^{2}+o(1),
\end{gathered}
$$

and

$$
\left|x_{n}^{1}-x_{n}^{2}\right| \rightarrow \infty \text { as } n \rightarrow \infty .
$$

Indeed, if (2.19) is not true, then

$$
v_{n}^{2}\left(x+x_{n}^{2}\right)=v_{n}^{1}\left(x+x_{n}^{2}-x_{n}^{1}+x_{n}^{1}\right)+V^{2}(x),
$$

which implies that $V^{2}=0$ by $v_{n}^{1}\left(\cdot+x_{n}^{1}\right) \rightarrow 0$ and $v_{n}^{2}\left(\cdot+x_{n}^{2}\right) \rightarrow 0$ in $H^{2}$, a contradiction.

An argument of iteration and orthogonal extraction allows us to construct the families $\left\{x_{n}^{j}\right\}_{j=1}^{\infty}$ and $\left\{V^{j}\right\}_{j=1}^{\infty}$ satisfying the claim above. Furthermore, since the convergence of the series $\sum_{j=1}^{l}\left(\left\|V^{j}\right\|_{2}^{2}+\left\|\triangle V^{j}\right\|_{2}^{2}\right)$, we have

$$
\left\|V^{j}\right\|_{2}^{2}+\left\|\triangle V^{j}\right\|_{2}^{2} \rightarrow 0 \text { as } j \rightarrow \infty
$$

which implies that

$$
\eta\left(v_{n}^{j}\right) \leq 2\left(\left\|V^{j-1}\right\|_{2}+\left\|\Delta V^{j-1}\right\|_{2}\right) \rightarrow 0 \text { as } j \rightarrow \infty .
$$

Therefore, (2.7), (2.8) and (2.12) are true.

In the end, we shall prove that for $p \in\left(2, \frac{2 N}{(N-4)^{+}}\right)$

$$
\left\|v_{n}^{l}\right\|_{p} \rightarrow 0 \text { as } l \rightarrow \infty .
$$

Let $\chi_{R} \in S\left(R^{N}\right)$ such that supp $\hat{\chi_{R}}(\xi)=\left\{\frac{1}{2 R} \leq|\xi| \leq 2 R\right\}, \hat{\chi_{R}}=1$ on $\left\{\frac{1}{R} \leq|\xi| \leq\right.$ $R\}$, and $0 \leq \hat{\chi_{R}} \leq 1$ on supp $\hat{\chi_{R}}(\xi)$, where ${ }^{\wedge}$ denotes the Fourier transform. It is obvious that

$$
v_{n}^{l}=\chi_{R} * v_{n}^{l}+\left(\delta-\chi_{R}\right) * v_{n}^{l},
$$

where $*$ is the convolution and $\delta$ is the Dirac function. Using the the definition of $\chi_{R}$, we have

$$
\left\|\left(\delta-\chi_{R}\right) * v_{n}^{l}\right\|_{2} \quad \leq C\left(\int_{|\xi| \leq \frac{1}{R}}\left|\hat{v}_{n}^{l}(\xi)\right|^{2} d \xi+\int_{|\xi| \geq R}\left|\hat{v}_{n}^{l}(\xi)\right|^{2} d \xi\right)^{\frac{1}{2}} .
$$

Since $v_{n}^{l}$ is bounded in $L^{2}$, for any $n \geq 1$, we have

$$
\int_{|\xi| \leq \frac{1}{R}}\left|\hat{v}_{n}^{l}(\xi)\right|^{2} d \xi+\int_{|\xi| \geq R}\left|\hat{v}_{n}^{l}(\xi)\right|^{2} d \xi \rightarrow 0, \quad \text { as } \quad R \rightarrow \infty \text {. }
$$


Using the Sobolev embedding $\dot{H}^{2} \hookrightarrow L^{\frac{2 N}{(N-4)^{+}}}$, we have

$$
\begin{aligned}
& \left\|\left(\delta-\chi_{R}\right) * v_{n}^{l}\right\|_{L^{(N-4)^{+}}} \leq C\left\|\left(\delta-\chi_{R}\right) * v_{n}^{l}\right\|_{\dot{H}^{2}} \\
& \leq C\left(\int|\xi|^{4}\left|\left(1-\hat{\chi}_{R}(\xi)\right) \hat{v}_{n}^{l}(\xi)\right|^{2} d \xi\right)^{\frac{1}{2}} \\
& \leq C\left(\int_{|\xi| \leq \frac{1}{R}}|\xi|^{4}\left|\hat{v}_{n}^{l}(\xi)\right|^{2} d \xi+\int_{|\xi| \geq R}|\xi|^{4}\left|\hat{v}_{n}^{l}(\xi)\right|^{2} d \xi\right)^{\frac{1}{2}} .
\end{aligned}
$$

Since $v_{n}^{l}$ is bounded in $H^{2}$, for any $n \geq 1$, we have

$$
\int_{|\xi| \leq \frac{1}{R}}|\xi|^{4}\left|\hat{v}_{n}^{l}(\xi)\right|^{2} d \xi+\int_{|\xi| \geq R}|\xi|^{4}\left|\hat{v}_{n}^{l}(\xi)\right|^{2} d \xi \rightarrow 0, \quad \text { as } \quad R \rightarrow \infty .
$$

Taking $p \in\left(2, \frac{2 N}{(N-4)^{+}}\right)$and using the Hölder interpolation inequality and (2.26)-

(2.28), we have

$$
\begin{aligned}
\left\|\left(\delta-\chi_{R}\right) * v_{n}^{l}\right\|_{p} & \leq\left\|\left(\delta-\chi_{R}\right) * v_{n}^{l}\right\|_{2}^{\theta}\left\|\left(\delta-\chi_{R}\right) * v_{n}^{l}\right\|_{\frac{2 N}{(N-4)^{+}}}^{1-\theta} \\
& \rightarrow 0, \quad \text { as } R \rightarrow \infty
\end{aligned}
$$

where $\frac{1}{p}=\frac{\theta}{2}+\frac{1-\theta}{\frac{2 N}{(N-4)^{+}}}$and $0<\theta<1$.

On the other hand, by the definition of $\chi_{R}$ one can estimate

$$
\left\|\chi_{R} * v_{n}^{l}\right\|_{p} \leq C\left\|\chi_{R} * v_{n}^{l}\right\|_{\infty}^{\frac{p-2}{p}}\left\|\chi_{R} * v_{n}^{l}\right\|_{2}^{\frac{2}{p}} .
$$

In view of the definition of $\mu\left(v_{n}\right)$, we have

$$
\lim _{n \rightarrow \infty} \sup \left\|\chi_{R} * v_{n}^{l}\right\|_{\infty} \leq \sup \left\{\left|\int \chi_{R}(-x) V(x) d x\right|, V \in \mu\left(v_{n}\right)\right\} .
$$

Using the Parseval identity and Hölder inequality, we have

$$
\begin{aligned}
\left|\int \chi_{R}(-x) V(x) d x\right| & =\left|\int \mathcal{F}^{-1}\left[\chi_{R}(-x)\right] \mathcal{F}[V(x)] d \xi\right| \\
& =\left|\int \widehat{\chi_{R}}(\xi) \widehat{V}(\xi) d \xi\right| \\
& \leq C \int_{\frac{1}{2 R} \leq|\xi| \leq 2 R} \frac{1}{|\xi|^{2}}|\xi|^{2}|\widehat{V}(\xi)| d \xi \\
& \leq C R^{N+2}\|\triangle V\|_{2} \leq C R^{N+2} \eta\left(v_{n}^{l}\right) .
\end{aligned}
$$

Taking $R=\left(\frac{1}{\eta\left(v_{n}^{l}\right)}\right)^{\frac{1}{N+2}-\varepsilon}$ with $\forall \varepsilon>0$ sufficient small, we have

$$
\left|\int \chi_{R}(-x) V(x) d x\right| \rightarrow 0 \text { as } l \rightarrow \infty .
$$

It follows from (2.30), 2.31) and (2.33) that for $p \in\left(2, \frac{2 N}{(N-4)^{+}}\right)$,

$$
\left\|\chi_{R} * v_{n}^{l}\right\|_{p} \rightarrow 0 \text { as } l \rightarrow \infty .
$$

Applying (2.24), (2.29) and (2.34), we have

$$
\lim _{n \rightarrow \infty} \sup \left\|v_{n}^{l}\right\|_{p} \rightarrow 0, \text { as } l \rightarrow \infty .
$$

This completes the proof. 


\section{Variational Structure}

In order to study the variational structure of the ground state, we consider the following elliptic equation

$$
\triangle^{2} Q+Q-|Q|^{\frac{8}{N}} Q=0, \quad Q \in H^{2} .
$$

Define the variational problem

$$
J:=\min \left\{J(u): u \in H^{2}\right\}, \text { where } J(u):=\frac{\left(\int|u|^{2} d x\right)^{\frac{4}{N}}\left(\int|\triangle u|^{2} d x\right)}{\int|u|^{2+\frac{8}{N}} d x} .
$$

By some basic calculations, it is easy to check that if $W$ is the minimizer of $J(u)$, we have the following lemma, one can also see 6 16. But we provide the detail here for the reader's convenience.

Lemma 3.1. If $W$ is the minimizer of $J(u)$, then $W$ satisfies

$$
\|W\|_{2}^{\frac{8}{N}} \triangle^{2} W+\frac{4}{N}\|\triangle W\|_{2}^{2}\|W\|_{2}^{\frac{8}{N}-2} W-J\left(1+\frac{4}{N}\right)|W|^{\frac{8}{N}} W=0 .
$$

Proof. It follows from the fact that $W$ is a minimizing function of $J(u)$ in $H^{2}$, and we have $\forall v \in C_{0}^{\infty}\left(R^{N}\right)$

$$
\left.\frac{d}{d \varepsilon} J(W+\varepsilon v)\right|_{\varepsilon=0}=0 .
$$

By some computations, we have

$$
\begin{gathered}
\left.\frac{d}{d \varepsilon}\left\{\|(W+\varepsilon v)\|_{2}^{\frac{8}{N}}\right\}\right|_{\varepsilon=0}=\frac{4}{N}\|W\|_{2}^{\frac{8}{N}-2} \int 2 \Re W \bar{v} d x, \\
\left.\frac{d}{d \varepsilon}\left\{\|\triangle(W+\varepsilon v)\|_{2}^{2}\right\}\right|_{\varepsilon=0}=\int 2 \Re \triangle^{2} W \bar{v} d x
\end{gathered}
$$

and

$$
\left.\frac{d}{d \varepsilon}\left\{\|W+\varepsilon v\|_{2+\frac{8}{N}}^{2+\frac{8}{N}}\right\}\right|_{\varepsilon=0}=\left(1+\frac{4}{N}\right) 2 \Re \int|W|^{\frac{8}{N}} W \bar{v} d x .
$$

By (3.4)-(3.7), we have

$$
\begin{aligned}
& \frac{4}{N}\|W\|_{2+\frac{8}{N}}^{2+\frac{8}{N}}\|\triangle W\|_{2}^{2}\|W\|_{2}^{\frac{8}{N}-2} \int 2 \Re W \bar{v} d x+\|W\|_{2+\frac{8}{N}}^{2+\frac{8}{N}}\|W\|_{2}^{\frac{8}{N}} \int 2 \Re \triangle^{2} W \bar{v} d x \\
= & \left(1+\frac{4}{N}\right)\|\triangle W\|_{2}^{2}\|W\|_{2}^{\frac{8}{N}} 2 \Re \int|W|^{\frac{8}{N}} W \bar{v} d x,
\end{aligned}
$$

which implies that (3.3) is true.

Now, we use the profile decomposition of the bounded sequence in $H^{2}$ to obtain the following proposition.

Proposition 3.2. $J$ is attained at a function $U(x) \in H^{2}$ with the following properties:

$$
U(x)=a Q\left(\lambda x+x_{0}\right) \text { for some } a \in \mathcal{C}^{*}, \lambda>0 \text { and } x_{0} \in R^{N}
$$

where $Q$ is the solution of ground state elliptic Equation (3.1). Moreover,

$$
J=\frac{1}{1+\frac{4}{N}}\|Q\|_{2}^{\frac{8}{N}} .
$$


Proof. If we set $u^{\lambda, \mu}=\mu u(\lambda x)$, where $\mu=\frac{\|u\|_{2}^{\frac{N}{4}-1}}{\|\Delta u\|_{2}^{\frac{N}{4}}}$ and $\lambda=\frac{\|u\|_{2}^{\frac{1}{2}}}{\|\Delta u\|_{2}^{\frac{1}{2}}}$, we have

$$
\left\|u^{\lambda, \mu}\right\|_{2}=1,\left\|\triangle u^{\lambda, \mu}\right\|_{2}=1 \text { and } J\left(u^{\lambda, \mu}\right)=J(u) .
$$

Now, choosing a minimizing sequence $\left\{u_{n}\right\}_{n=1}^{\infty} \subset H^{2}$ such that $J\left(u_{n}\right) \rightarrow J$ as $n \rightarrow \infty$, after scaling, we may assume

$$
\left\|u_{n}\right\|_{2}=1 \text { and }\left\|\triangle u_{n}\right\|_{2}=1 \text {, }
$$

and we have

$$
J\left(u_{n}\right)=\frac{1}{\int\left|u_{n}\right|^{2+\frac{8}{N}} d x} \rightarrow J, \text { as } n \rightarrow \infty .
$$

Note that $\left\{u_{n}\right\}_{n=1}^{\infty}$ is bounded in $H^{2}$. It follows form the profile decomposition (Proposition 2.3)that

$$
u_{n}(x)=\sum_{j=1}^{l} U^{j}\left(x-x_{n}^{j}\right)+r_{n}^{l}(x)
$$

and

$$
\sum_{j=1}^{l}\left\|U_{n}^{j}\right\|_{2}^{2} \leq 1, \quad \sum_{j=1}^{l}\left\|\triangle U_{n}^{j}\right\|_{2}^{2} \leq 1,
$$

where $U_{n}^{j}=U^{j}\left(x-x_{n}^{j}\right)$. Moreover, since $2<2+\frac{8}{N}<\frac{2 N}{(N-4)^{+}}$, for $r_{n}^{l}$, we have

$$
\int\left|r_{n}^{l}\right|^{2+\frac{8}{N}} d x \rightarrow 0, \text { as } l \rightarrow+\infty
$$

Using the orthogonal conditions and the following elementary inequality $(p>1)$

$$
\left.|| \sum_{j=1}^{l} a_{j}\right|^{1+p}-\left.\sum_{j=1}^{l}\left|a_{j}\right|^{1+p}\left|\leq C \sum_{j \neq k}\right| a_{j}|| a_{k}\right|^{p},
$$

we have

$$
\int\left|\sum_{j=1}^{l} U^{j}\left(x-x_{n}^{j}\right)\right|^{2+\frac{8}{N}} d x \rightarrow \sum_{j=1}^{l} \int\left|U^{j}\right|^{2+\frac{8}{N}} d x, \text { as } n \rightarrow \infty .
$$

Therefore, by (3.13), (3.15) and (3.17), we have

$$
\sum_{j=1}^{l} \int\left|U^{j}\right|^{2+\frac{8}{N}} d x \rightarrow \frac{1}{J}, \text { as } n \rightarrow \infty .
$$

For another thing, by the definition of $J$, we have

$$
J \int\left|U^{j}\right|^{2+\frac{8}{N}} d x \leq\left\|U^{j}\right\|_{2}^{\frac{8}{N}}\left\|\triangle U^{j}\right\|_{2}^{2} .
$$

Since the series $\sum_{j}\left\|U^{j}\right\|_{2}^{2}$ is convergent, there exists a $j_{0} \geq 1$ such that

$$
\left\|U^{j_{0}}\right\|_{2}=\sup \left\{\left\|U^{j}\right\|_{2} \mid j \geq 1\right\} .
$$


It follows from (3.18)-(3.20) that

$$
\begin{aligned}
1 \leq J\left(\sum_{j=1}^{l} \int\left|U^{j}\right|^{2+\frac{8}{N}} d x\right) & \leq \sup \left\{\left\|U^{j}\right\|_{2}^{\frac{8}{N}} \mid j \geq 1\right\}\left(\sum_{j=1}^{l}\left\|\nabla U^{j}\right\|_{2}^{2}\right) \\
& \leq\left\|U^{j_{0}}\right\|_{2}^{\frac{8}{N}}\left(\sum_{j=1}^{l}\left\|\nabla U^{j}\right\|_{2}^{2}\right) \leq\left\|U^{j_{0}}\right\|_{2}^{\frac{8}{N}} .
\end{aligned}
$$

It follows from (3.14) that $\left\|U^{j_{0}}\right\|_{2}=1$, which implies that there exists only one term $U^{j_{0}} \neq 0$ such that

$$
\left\|U^{j_{0}}\right\|_{2}=1,\left\|\triangle U^{j_{0}}\right\|_{2}=1, \text { and } \int\left|U^{j_{0}}\right|^{2+\frac{8}{N}} d x=\frac{1}{J} .
$$

Therefore, we show that $U^{j_{0}}$ is the minimizer of $J(u)$. It follows from Lemma 3.1 that

$$
\triangle^{2} U^{j_{0}}+\frac{4}{N} U^{j_{0}}-\left(1+\frac{4}{N}\right) J\left|U^{j_{0}}\right|^{\frac{8}{N}} U^{j_{0}}=0 .
$$

We take $U^{j_{0}}=a Q\left(\lambda x+x_{0}\right)$ for the reason of symmetric invariance of Equation (3.1), where $a \in C^{*}, \lambda>0, x_{0} \in R^{N}$ and $Q$ is the solution of (3.1).

On the other hand, if $Q$ is the solution of Equation (3.1), we claim

$$
\int|\triangle Q|^{2} d x+\int|Q|^{2} d x-\int Q^{2+\frac{8}{N}} d x=0
$$

and

$$
\left(2-\frac{N}{2}\right) \int|\triangle Q|^{2} d x-\frac{N}{2} \int|Q|^{2} d x+\frac{N}{2+\frac{8}{N}} \int Q^{2+\frac{8}{N}} d x=0 .
$$

Indeed, Multiplying (3.1) by $Q$ and integrating by parts, we have that (3.24) is true.

Multiplying (3.1) by $x \cdot \nabla Q$ and integrating by parts, we have

$$
\int \triangle^{2} Q x \cdot \nabla Q d x+\int Q x \cdot \nabla Q d x-\int|Q|^{\frac{8}{N}} Q x \cdot \nabla Q d x=0 .
$$

For another thing, we have

$$
\begin{gathered}
\int \triangle^{2} Q x \cdot \nabla Q d x=2 \int|\triangle Q|^{2} d x+\int x \cdot \nabla\left(\frac{|\triangle Q|^{2}}{2}\right) d x=\left(2-\frac{N}{2}\right) \int|\triangle Q|^{2} d x, \\
\int Q x \cdot \nabla Q d x=-\frac{N}{2} \int Q^{2} d x
\end{gathered}
$$

and

$$
\int|Q|^{\frac{8}{N}} Q x \cdot \nabla Q d x=-\frac{N}{2+\frac{8}{N}} \int Q^{2+\frac{8}{N}} d x .
$$

Collecting the above identities, we have that (3.25) is true.

Now, we return to the proof of Proposition 3.2. By some computations, we have that $\left\|U^{j_{0}}\right\|_{2}^{2}=\frac{|a|^{2}}{\lambda^{N}}\|Q\|_{2}^{2}=1,\left\|\triangle U^{j_{0}}\right\|_{2}^{2}=\frac{|a|^{2}}{\lambda^{N-4}}\|\triangle Q\|_{2}^{2}=1$ and $\int\left|U^{j_{0}}\right|^{2+\frac{8}{N}} d x=$ $\frac{|a|^{2+\frac{8}{N}}}{\lambda^{N}} \int|Q|^{2+\frac{8}{N}} d x=\frac{1}{J}$. Applying Claim (3.24) and (3.25), we have

$$
\frac{1}{1+\frac{4}{N}} \int|Q|^{2+\frac{8}{N}} d x=\int|\triangle Q|^{2} d x
$$


which implies that

$$
J=\frac{\lambda^{N}}{|a|^{2+\frac{8}{N}}} \frac{1}{\int|Q|^{2+\frac{8}{N}} d x}=\frac{1}{1+\frac{4}{N}}\|Q\|_{2}^{\frac{8}{N}} .
$$

This completes the proof Proposition 3.2.

Remark 3.3. In [6], Fibich etal also showed the following sharp GargliardoNirenberg inequality

$$
\frac{1}{1+\frac{4}{N}} \int|u|^{2+\frac{8}{N}} d x \leq \frac{1}{\left(\int|Q|^{2} d x\right)^{\frac{4}{N}}}\left(\int|u|^{2} d x\right)^{\frac{4}{N}}\left(\int|\triangle u|^{2} d x\right),
$$

where $Q$ is the solution of ground state Equation (3.1), but the existence of the ground state Equation [3.1) is not addressed in their paper. In this paper, we prove the existence of the ground state Equation (3.1). Our results are more strong than Fibich etal's results and the methods are different.

At the end of this section, we prove Theorem 1.1 by applying the profile decomposition of the bounded sequence in $H^{2}$ and the sharp Gagliardo-Nirenberg inequality (Proposition 3.2).

Proof of Theorem 1.1. By extracting a subsequence, we may replace lim sup in the assumption in Theorem 1.1 by lim. According to the profile decomposition in Proposition 2.3, the sequence $\left\{v_{n}\right\}_{n=1}^{\infty}$ can be written up to a subsequence, as

$$
v_{n}(x)=\sum_{j=1}^{l} V^{j}\left(x-x_{n}^{j}\right)+v_{n}^{l}(x)
$$

with

$$
\lim _{l \rightarrow \infty} \lim _{n \rightarrow \infty} \sup \left\|v_{n}^{l}\right\|_{p}=0,
$$

for $p \in\left(2, \frac{2 N}{(N-4)^{+}}\right)$, and we have the following estimations

$$
\begin{gathered}
\left\|v_{n}\right\|_{2}^{2}=\sum_{j=1}^{l}\left\|V^{j}\right\|_{2}^{2}+\left\|v_{n}^{l}\right\|_{2}^{2}+o(1), \\
\left\|\triangle v_{n}\right\|_{2}^{2}=\sum_{j=1}^{l}\left\|\triangle V^{j}\right\|_{2}^{2}+\left\|\triangle v_{n}^{l}\right\|_{2}^{2}+o(1) .
\end{gathered}
$$

This implies that

$$
\begin{aligned}
m^{2+\frac{8}{N}} & \leq \lim _{n \rightarrow \infty} \sup \left\|v_{n}\right\|_{2+\frac{8}{N}}^{2+\frac{8}{N}} \\
& \leq \lim _{n \rightarrow \infty} \sup \left\|\sum_{j=1}^{l} V^{j}\left(x-x_{n}^{j}\right)+v_{n}^{l}(x)\right\|_{2+\frac{8}{N}}^{2+\frac{8}{N}} \\
& \leq \lim _{n \rightarrow \infty} \sup \left(\left\|\sum_{j=1}^{l} V^{j}\left(x-x_{n}^{j}\right)\right\|_{2+\frac{8}{N}}+\left\|v_{n}^{l}(x)\right\|_{2+\frac{8}{N}}\right)^{2+\frac{8}{N}} \\
& \leq\left\|\sum_{j=1}^{l} V^{j}\left(x-x_{n}^{j}\right)\right\|_{2+\frac{8}{N}}^{2+\frac{8}{N}} \quad \text { as } l \rightarrow \infty .
\end{aligned}
$$


Using the elementary inequality

$$
\left.|| \sum_{j=1}^{l} a_{j}\right|^{2+\frac{8}{N}}-\left.\sum_{j=1}^{l}\left|a_{j}\right|^{2+\frac{8}{N}}\left|\leq C \sum_{j \neq k}\right| a_{j}|| a_{k}\right|^{1+\frac{8}{N}},
$$

and the pairwise orthogonality of the family $\left\{x_{n}^{j}\right\}_{j=1}^{\infty}$, we have that the mixed terms in (3.30) vanish. Hence, we have

$$
m^{2+\frac{8}{N}} \leq \sum_{j=1}^{\infty}\left\|V^{j}\right\|_{2+\frac{8}{N}}^{2+\frac{8}{N}} .
$$

On the other hand, using the Gagliardo-Nirenberg inequality, we have

$$
\sum_{j=1}^{\infty}\left\|V^{j}\right\|_{2+\frac{8}{N}}^{2+\frac{8}{N}} \leq \frac{1+\frac{4}{N}}{\|Q\|_{2}^{\frac{8}{N}}} \sup \left\{\left\|V^{j}\right\|_{2}^{\frac{8}{N}}, j \geq 1\right\}\left(\sum_{j=1}^{\infty}\left\|\Delta V^{j}\right\|_{2}^{2}\right) .
$$

By (1.8) and (3.29), we have

$$
\sum_{j=1}^{\infty}\left\|\triangle V^{j}\right\|_{2}^{2} \leq \lim _{n \rightarrow \infty} \sup \left\|\triangle v_{n}\right\|_{2}^{2} \leq M^{2}
$$

Therefore, we have

$$
\sup _{j \geq 1}\left\|V^{j}\right\|_{2}^{\frac{8}{N}} \geq \frac{\|Q\|_{2}^{\frac{8}{N}} m^{2+\frac{8}{N}}}{\left(1+\frac{4}{N}\right) M^{2}} .
$$

Since the series $\sum_{j=1}^{\infty}\left\|V^{j}\right\|_{2}^{2}$ is convergent, we have that the supremum of $\left\{\left\|V^{j}\right\|_{2}^{\frac{8}{N}} ; j \geq\right.$ $1\}$ is attained. In particular, there exists a $j_{0} \geq 1$ such that

$$
\left\|V^{j_{0}}\right\|_{2}^{\frac{8}{N}} \geq \frac{\|Q\|_{2}^{\frac{8}{N}} m^{2+\frac{8}{N}}}{\left(1+\frac{4}{N}\right) M^{2}}
$$

By a change of variables, we have

$$
v_{n}\left(x+x_{n}^{j_{0}}\right)=V^{j_{0}}(x)+\sum_{j \neq j_{0}} V^{j}\left(x-x_{n}^{j}+x_{n}^{j_{0}}\right)+\tilde{v}_{n}^{l}(x),
$$

where $\tilde{v}_{n}^{l}(x)=v_{n}^{l}\left(x+x_{n}^{j_{0}}\right)$. Applying the pairwise orthogonality of the family $x_{n}^{j}$ to (3.37), we have

$$
V^{j}\left(x-x_{n}^{j}+x_{n}^{j_{0}}\right) \rightarrow 0 \text { weakly in } H^{2}
$$

for $j \neq j_{0}$. Hence, we have

$$
v_{n}\left(x+x_{n}^{j_{0}}\right) \rightarrow V^{j_{0}}+\tilde{v}^{l} .
$$

where $\tilde{v}^{l}$ denote the weak limit of $\tilde{v}_{n}^{l}$. Using the Proposition 2.3, we have

$$
\left\|\tilde{v}^{l}\right\|_{2+\frac{8}{N}} \leq \lim _{n \rightarrow \infty} \sup \left\|\tilde{v}_{n}^{l}\right\|_{2+\frac{8}{N}}=\lim _{n \rightarrow \infty} \sup \left\|v_{n}^{l}\right\|_{2+\frac{8}{N}} \rightarrow 0, \text { as } l \rightarrow \infty,
$$

which implies that

$$
\tilde{v}^{l}=0 \quad \text { for } l \geq j_{0},
$$

by the uniqueness of the weak limit.

Therefore, we have

$$
v_{n}\left(x+x_{n}^{j_{0}}\right) \rightarrow V^{j_{0}} \quad \text { weakly in } H^{2},
$$


which implies the sequence $x_{n}^{j_{0}}$ and the function $V^{j_{0}}$ now fulfill the condition of Theorem 1.1. This completes the proof.

\section{4. $L^{2}$-Concentration}

In this section, we shall use the compactness results in Theorem 3.4 to study the $L^{2}$-concentration properties of blow-up solutions to the Cauchy problem (1.1)(1.2) in the general case. This result extends the results in $\mathbf{1}$ to the non-radially symmetric blow-up solutions. More precisely, we have the following theorem.

Theorem 4.1. Let $u(t, x) \in C\left([0, T) ; H^{2}\right)$ be the corresponding blow-up solution of the Cauchy problem 1.1)-(1.2) such that

$$
\lim _{t \rightarrow T}\|\triangle u(t, x)\|_{2}=+\infty \text {. }
$$

Suppose that $a(t)>0$ is any function such that $a(t)\|\Delta u(t)\|_{2}^{\frac{1}{2}} \rightarrow+\infty$ as $t \rightarrow T$. Then, there exists $y(t) \in R^{N}$ such that

$$
\lim _{t \rightarrow T} \inf \int_{|x-y(t)| \leq a(t)}|u(t, x)|^{2} d x \geq \int|Q|^{2} d x,
$$

where $Q$ is the solution of ground state Equation (3.1).

Proof. Since $u(t, x) \in C\left([0, T) ; H^{2}\right)$ is the corresponding blow-up solution of the Cauchy problem (1.1)-(1.2) such that $\lim _{t \rightarrow T}\|\triangle u(t, x)\|_{2}=+\infty$. For any $t_{k} \rightarrow T$ as $k \rightarrow+\infty$, we take

$$
\frac{1}{\lambda_{k}^{2}}=\left\|\triangle u\left(x, t_{k}\right)\right\|_{2} \rightarrow+\infty, \quad \text { as } \mathrm{k} \rightarrow+\infty .
$$

Considering $U_{k}=\lambda_{k}^{\frac{N}{2}} u\left(\lambda_{k} x\right)$, by direct computations, we have

$$
\begin{cases}\left\|U_{k}\right\|_{2} & =\left\|u\left(t_{k}\right)\right\|_{2}=\left\|u_{0}\right\|_{2} \\ \left\|\triangle U_{k}\right\|_{2} & =\lambda_{k}^{2}\left\|\triangle u\left(t_{k}\right)\right\|_{2}=1\end{cases}
$$

Therefore, $U_{k}$ is a uniformly bounded sequence in $H^{2}$ by (4.4). Note that

$$
\begin{aligned}
E\left(U_{k}\right) & =\frac{1}{2} \int\left|\triangle U_{k}\right|^{2} d x-\frac{1}{2+\frac{8}{N}} \int\left|U_{k}\right|^{2+\frac{8}{N}} d x \\
& =\lambda_{k}^{4} E\left(u_{0}\right) \\
& \rightarrow 0, \text { as } k \rightarrow+\infty,
\end{aligned}
$$

by the conservation of energy. Combining (4.4) with (4.5), we have

$$
\lim _{k \rightarrow \infty} \int\left|U_{k}\right|^{2+\frac{8}{N}} d x \geq 1+\frac{4}{N} \text {. }
$$

Applying Theorem 1.1 to the sequence $U_{k}$ (with $M=1, m^{2+\frac{8}{N}}=1+\frac{4}{N}$ ), we have that there exists $\left\{y_{k}\right\} \subset R^{N}$

$$
U_{k}\left(x+y_{k}\right) \rightarrow U(x) \text { weakly in } H^{2}, \text { with }\|U\|_{2} \geq\|Q\|_{2},
$$

where $Q$ is the ground state solution of Equation (3.1). That is,

$$
\lambda_{k}^{\frac{N}{2}} u\left(t_{k}, \lambda_{k}\left(x+y_{k}\right)\right) \rightarrow U(x) \text { weakly in } H^{2},
$$


which implies that for every $A>0$

$$
\lim _{k \rightarrow \infty} \inf \int_{|x| \leq A} \lambda_{k}^{N}\left|u\left(t_{k}, \lambda_{k}\left(x+y_{k}\right)\right)\right|^{2} d x \geq \int_{|x| \leq A}|U|^{2} d x .
$$

Since the assumption $\frac{a\left(t_{k}\right)}{\lambda\left(t_{k}\right)} \rightarrow+\infty$ as $k \rightarrow+\infty$, we have

$$
\lim _{k \rightarrow \infty} \inf \sup _{y \in R^{N}} \int_{|x-y| \leq a\left(t_{k}\right)}\left|u\left(t_{k}, x\right)\right|^{2} d x \geq \int_{|x| \leq A}|U|^{2} d x .
$$

For every $A>0$, we have

$$
\lim _{k \rightarrow \infty} \inf \sup _{y \in R^{N}} \int_{|x-y| \leq a\left(t_{k}\right)}\left|u\left(t_{k}, x\right)\right|^{2} d x \geq \int|U|^{2} d x .
$$

Therefore, since the sequence $\left\{t_{k}\right\}$ is arbitrary and (4.7), we have

$$
\lim _{t \rightarrow T} \inf \sup _{y \in R^{N}} \int_{|x-y| \leq a(t)}|u(t, x)|^{2} d x \geq \int|Q|^{2} d x .
$$

On the other hand, for every $t \in[0, T)$, the function $y \mapsto \int_{|x-y| \leq a(t)}|u(t, x)|^{2} d x$ is continuous and goes to 0 at infinity, and we have

$$
\sup _{y \in R^{N}} \int_{|x-y| \leq a(t)}|u(t, x)|^{2} d x=\int_{|x-y(t)| \leq a(t)}|u(t, x)|^{2} d x,
$$

for some $y(t) \in R^{N}$. This completes the proof.

Applying the lower blow-up rate of the solutions to the Cauchy problem (1.1)(1.2) obtained by Baruch, Fibich and Mandelbaum 1 (see Lemma 2.2), we have the following rate of $L^{2}$-concentration of the blow-up solutions to the Cauchy problem (1.1)-(1.2) (see Theorem 1.2). At the end of this section, we give the proof of Theorem 1.2

Proof of Theorem 1.2. The result follows immediately from Lemma 2.2 and Theorem 4.1.

\section{Limiting Profile of Minimal Mass Blow-up Solutions}

In this section, we assume that the ground state solution of Equation (3.1) is unique up to translations in space, phase and dilations, which is also denoted by $Q(x)$, where it is assumed as the same as in 33 for the classical Schrödinger equation (1.4). Using the compactness lemma obtained in Section 3 and the characterization of the corresponding ground state, we obtain the limiting profile of the blow-up solutions in $H^{2}$ for the Cauchy problem (1.1)-(1.2). More precisely, we obtain that if the initial data $u_{0} \in H^{2}$ satisfies $\left\|u_{0}\right\|_{2}=\|Q(x)\|_{2}$, then the corresponding blow-up solution of the Cauchy problem (1.1)-(1.2) $u(t, x)$ remains close to $Q(x)$ in $H^{2}$ up to scaling and phase parameters, and also translation in the nonradial case. At first, we consider the variational characterization of the ground state of Equation (3.1), as follows.

Lemma 5.1. If $u \in H^{2}$ is such that $\|u\|_{2}=\|Q\|_{2}$ and $E(u)=0$, then $u(x)$ is of the following form

$$
u(x)=e^{i \gamma} \lambda^{\frac{N}{2}} Q\left(\lambda x+x_{0}\right), \text { for some } \gamma \in R, \lambda>0, x_{0} \in R^{N},
$$

where $Q$ is the unique solution of ground state Equation (3.1). 
Proof. Since $E(u)=0$, we have

$$
\int|\triangle u|^{2} d x=\frac{1}{1+\frac{4}{N}} \int|u|^{2+\frac{8}{N}} d x
$$

Hence, we have

$$
J(u)=\frac{\left(\int|u|^{2} d x\right)^{\frac{4}{N}}\left(\int|\triangle u|^{2} d x\right)}{\int|u|^{2+\frac{8}{N}} d x}=\frac{1}{1+\frac{4}{N}}\|u\|_{2}^{\frac{8}{N}}=\frac{1}{1+\frac{4}{N}}\|Q\|_{2}^{\frac{8}{N}}=J,
$$

which implies that $u$ is a minimizer of $J(u)$. By Proposition 3.2 and the uniqueness of $Q$, we have that $u$ is of the form $u(x)=a Q\left(\lambda x+x_{0}\right)$. On the other hand, by $\|u\|_{2}=\|Q\|_{2}$, we have $|a|=\lambda^{\frac{N}{2}}$. Therefore, since the value of $u(x)$ is in $\mathcal{C}$, there exists $\gamma \in R$ such that

$$
u(x)=e^{i \gamma} \lambda^{\frac{N}{2}} Q\left(\lambda x+x_{0}\right),
$$

where $\lambda>0, x_{0} \in R^{N}$ and $Q$ is the unique ground state solution of Equation (3.1). This completes the proof.

Now, we are in proposition to prove Theorem 1.3 by applying the variational characteristic of the ground state of Equation (3.1).

Proof of Theorem 1.3. We show that for any sequence $t_{k} \rightarrow T$, there is a subsequence $t_{k_{j}}, y_{k_{j}}$ and $\gamma\left(t_{k_{j}}\right)$ such that

$$
\lambda^{\frac{N}{2}}\left(t_{k_{j}}\right) u\left(t_{k_{j}}, \lambda\left(t_{k_{j}}\right)\left(x+y\left(t_{k_{j}}\right)\right)\right) e^{i \gamma\left(t_{k_{j}}\right)} \rightarrow Q(x) \text { strongly in } H^{2} \text { as } j \rightarrow \infty,
$$

where $Q(x)$ is the unique ground state solution of Equation (3.1). If not, then (5.4) does not holds along some sequence $t_{k_{r}}$. But then we can find a subsequence of $t_{k_{r}}$ along with (5.4) holds, this is a contradiction. Since $t_{k}$ is an arbitrary sequence approaching $T$, and (1.12) follows.

Since $u(t, x) \in C\left([0, T) ; H^{2}\right)$ is the blow-up solution of the Cauchy problem (1.1)-(1.2), there is a $0<T<+\infty$ such that $\lim _{t \rightarrow T}\|\Delta u\|_{2}=+\infty$. For any $t_{k} \rightarrow T$ as $k \rightarrow+\infty$, we take

$$
\frac{1}{\lambda_{k}^{2}}=\left\|\triangle u\left(x, t_{k}\right)\right\|_{2} \rightarrow+\infty, \text { as } \mathrm{k} \rightarrow+\infty .
$$

Consider $U_{k}=\lambda_{k}^{\frac{N}{2}} u\left(\lambda_{k} x\right)$, by direct computations, we have

$$
\left\{\begin{array}{l}
\left\|U_{k}\right\|_{2}=\left\|u\left(t_{k}\right)\right\|_{2}=\left\|u_{0}\right\|_{2}=\|Q(x)\|_{2}, \\
\left\|\triangle U_{k}\right\|_{2}=\lambda_{k}^{2}\left\|\triangle u\left(t_{k}\right)\right\|_{2}=1 .
\end{array}\right.
$$

Therefore, $U_{k}$ is a uniformly bounded sequence in $H^{2}$ and $U_{k}$ has a weakly convergent subsequence $U_{k}$ (still denoted by $U_{k}$ ). Note that

$$
\begin{aligned}
E\left(U_{k}\right) & =\frac{1}{2}\left\|\triangle U_{k}\right\|_{2}^{2}-\frac{1}{2+\frac{8}{N}}\left\|U_{k}\right\|_{2+\frac{8}{N}}^{2+\frac{8}{N}} \\
& =\lambda_{k}^{4} E\left(u_{0}\right) \rightarrow 0, \text { as } k \rightarrow+\infty .
\end{aligned}
$$

Combining (5.6) with (5.7), one has

$$
\lim _{k \rightarrow \infty}\left\|U_{k}\right\|_{2+\frac{8}{N}}^{2+\frac{8}{N}}=1+\frac{4}{N} .
$$


Applying Theorem 1.1 to the sequence $U_{k}\left(M=1, m^{2+8 / N}=1+\frac{4}{N}\right)$, one has that there exist $\left\{y_{k}\right\} \subset R^{N}$ and $U \in H^{2}$ such that

$$
U_{k}\left(x+y_{k}\right) \rightarrow U(x) \text { weakly in } H^{2}
$$

with $\|U\|_{2} \geq\|Q\|_{2}$. Since $\|U\|_{2} \leq\left\|U_{k}\left(x+y_{k}\right)\right\|_{2}=\|Q\|_{2}$ and the Brézis-Lieb Lemma, one has

$$
U_{k}\left(x+y_{k}\right) \rightarrow U(x) \text { strongly in } L^{2} .
$$

By the Gagliardo-Nirenberg's inequality (see Proposition 3.2), there exists $\gamma_{k} \in R$ such that

$$
\left\|U_{k}\left(x+y_{k}\right) e^{i \gamma_{k}}-U\right\|_{2+\frac{8}{N}}^{2+\frac{8}{N}} \leq C\left\|U_{k}\left(x+y_{k}\right) e^{i \gamma_{k}}-U\right\|_{2}^{\frac{8}{N}}\left\|\triangle\left(U_{k}\left(x+y_{k}\right) e^{i \gamma_{k}}-U\right)\right\|_{2}^{2} .
$$

It follows from (5.6) and $\left\|\triangle U_{k}\left(x+y_{k}\right)\right\|_{2} \leq C$ that

$$
U_{k}\left(x+y_{k}\right) e^{i \gamma_{k}} \rightarrow U \text { strongly in } L^{2+\frac{8}{N}} .
$$

Next, we shall show that $U_{k}\left(x+y_{k}\right) e^{i \gamma_{k}}$ converges to $U$ strongly in $H^{2}$. We need only now show that $\|\triangle U\|_{2}=1$ by the Brézis-Lieb Lemma. Note that

$$
\begin{aligned}
0 & =\lim _{k \rightarrow \infty} E\left(U_{k} e^{i \gamma_{k}}\right) \\
& =\frac{1}{2}-\frac{1}{2+\frac{8}{N}} \lim _{k \rightarrow \infty}\left\|U_{k}\right\|_{2+\frac{8}{N}}^{2+\frac{8}{N}} \\
& =\frac{1}{2}-\frac{1}{2+\frac{8}{N}}\|U\|_{2+\frac{8}{N}}^{2+\frac{8}{N}} .
\end{aligned}
$$

Therefore, we have $\|\triangle U\|_{2}=1$ and the fact $U \not \equiv 0$, which implies that $U_{k}\left(x+y_{k}\right) e^{i \gamma_{k}}$ converges strongly to $U$ in $H^{2}$.

Therefore, applying the variational characteristic of the ground state of Equation(3.1), we have, $\exists y \in R^{N}, \gamma \in R$ such that $U(x)=Q(x+y) e^{i \gamma}$, which implies that

$$
\lambda_{k}^{\frac{N}{2}} u\left(t_{k}, \lambda_{k}\left(x+y_{k}\right)\right) e^{i \gamma_{k}} \rightarrow Q(x+y) e^{i \gamma} \text { strongly in } H^{2} \text { as } k \rightarrow \infty .
$$

By redefining the sequences $y_{k}$ and $\gamma_{k}$, we have (5.4) is true. This completes the proof.

Acknowledgement We thank the referee for pointing out some misprints and helpful suggestions.

\section{References}

[1] G. Baruch, G. Fibich, E. Mandelbaum, Singular solutions of the biharmonic Nonlinear Schrödinger equation, http://arxiv.org/abs/0912.1233v1.

[2] M. Ben-Artzi, H. Koch, and J.-C. Saut, Dispersion estimates for fourth order Schrödinger equations, C. R. Acad. Sci. Paris Ser. I Math., 330(2000), $87-92$.

[3] J. Bourgain, Refinements of Strichartz inequality and applications to 2dNLS with critical nonlinearity, Int. Math. Res. Not., (1998), 253-284.

[4] T. Cazenave, Semilinear Schrödinger equations, Courant Lecture Notes in Mathematics, 10, NYU, CIMS, AMS 2003. 
[5] J. Colliander, S. Raynor, C. Sulem and J. D. Wright, Ground state mass concentration in $L^{2}$-critical nonlinear Schrödinger equation below $H^{1}$, Math. Res. Lett., 12(2005), 357-375.

[6] G. Fibich, B. Ilan, G. Papanicolaou, Self-Focusing with Fourth-Order Dispersion, SIAM J. Appl. Math., 62(2002), 1437-1462.

[7] P. Gérard, Description du defaut de compacite de l'injection de Sobolev, ESAIM Control Optim. Calc. Var., 3(1998), 213-233.

[8] J. Ginibre and G. Velo, On a class of nonlinear Schrödinger equations I II, The Cauchy problem, general case, J. Funct. Anal., 32(1979), 1-71.

[9] T. Hmidi and S. Keraani, Blowup theory for the critical nonlinear Schrödinger equations revisited, Int. Math. Res. Not., 46(2005), 2815-2828.

[10] T. Hmidi and S. Keraani, Remarks on the blowup for the $L^{2}$-critical nonlinear Schrödinger equations, SIAM J. Math. Anal., 38(2006), 1035-1047.

[11] V. I. Karpman, Stabilization of soliton instabilities by higher-order dispersion: fourth order nonlinear Schrödinger-type equations, Phys. Rev. E, 53(1996), 1336-1339.

[12] V. I. Karpman, A. G. Shagalov, Stability of soliton described by nonlinear Schrödinger type equations with higher-order dispersion, Phys. D., 144(2000), 194-210.

[13] C. Kenig, G. Ponce, L. Vega, Oscillatory integrals and regularity of dispersive equations. Indiana Univ. Math. J., 40(1991), 33-69.

[14] R. Killip, T. Tao, M. Visan, The cubic nonlinear Schrödinger equation in two dimensions with radial data, J. Eur. Math. Soc., 11 (2009), 12031258.

[15] R. Killip, M. Visan, X. Zhang, The mass-critical nonlinear Schrödinger equation with radial data in dimensions three and higher, Anal. PDE, 1 (2008), 229-266.

[16] S. Levandosky, Stability and instability of fourth-order solitary waves, J. Dynam. Differential Equations, 10 (1998), no. 1, 151-188.

[17] F. Merle, Determination of blow-up solutions with minimal mass for nonlinear Schrödinger equations with critical power, Duke Math. J., 69(1993), 427-454.

[18] F. Merle and P. Raphaël, Blow-up dynamic and upper bound on the blow-up rate for critical nonlinear Schrödinger equation, Annals of Math., 16(2005), 157-222.

[19] F. Merle and P. Raphaël, On a sharp lower bound on the blow-up rate for the $L^{2}$-critical nonlinear Schrödinger equation, J. Amer. Math. Soc., 19(2006), 37-90.

[20] F. Merle and Y. Tsutsumi, $L^{2}$-concentration of blow up solutions for the nonlinear Schrödinger equation with critical power nonlinearity, J. Differential Equations, 84(1990), 205-214.

[21] C. Miao, G. Xu and L. Zhao, Global wellposedness and scattering for the focusing energycritical nonlinear Schrödinger equations of fourth order in the radial case. J. Differential Equations, 246 (2009), 3715-3749.

[22] C. Miao, G. Xu and L. Zhao, Global wellposedness and scattering for the defocusing energy-critical nonlinear Schrödinger equations of fourth order in dimensions $d \geq 9$, http://arxiv.org/abs/0807.0692v2. 
[23] H. Nawa, Asymptotic and limiting profiles of blowup solutions of the nonlinear Schrödinger equation with critical power, Commun. Pure Appl. Math., 52(1999), 193-270.

[24] B. Pausader, Global well-posedness for energy critical fourth-order Schrödinger equations in the radial case, Dynamics of PDE, 4 (2007), 197-225.

[25] B. Pausader, The cubic fourth-order Schrödinger equation, J. Funct. Anal., 256(2009),2473-2517.

[26] B. Pausader, The focusing energy-critical fourth-order Schrödinger equation with radial data, DCDS-A, to appear.

[27] B. Pausader and S. L. Shao, The mass-critical fourth-order Schrödinger equation in high dimensions, http://arxiv.org/abs/0906.1547v2.

[28] J. Segata, Modified wave operators for the fourth-order non-linear Schrödingertype equation with cubic non-linearity, Math. Meth. Appl. Sci., 26(2006), $1785-1800$.

[29] C. Sulem and P.-L. Sulem, The Nonlinear Schrödinger Equation. Selffocusing and Wave Collapse, Appl. Math. Sci. 139, Springer-Verlag, New York, 1999.

[30] Y. Tustusmi, Rate of $L^{2}$ concentration of blow-up solutions for the nonlinear Schrödinger equation with critical power, Nonlinear Anal. T. M. A., 15(1990), 719-724.

[31] N. Tzirakis, Mass concentration phenomenon for the quintic nonlinear Schrödinger equation in 1D, SIAM J. Math. Anal., 37(2006), 1923-1946.

[32] M. Visan, X. Zhang, On the blowup for the $L^{2}$-critical focusing nonlinear Schrödinger equation in higher dimensions below the energy class, SIAM J. Math. Anal., 39(2007), 34-56.

[33] M. I. Weinstein, Nonlinear Schrödinger equations and sharp interpolation estimates, Commun. Math. Phys., 87(1983), 567-576.

[34] M. I.Weinstein, On the structure and formation of singularities in solutions to nonlinear dispersive evolution equations, Commun. in PDE, 11(1986), $545-565$.

[35] M. I. Weinstein, The nonlinear Schrödinger equation-singularity formation, stability and dispersion. The connection between infinite-dimensional and finite-dimensional dynamical systems (Boulder, 1987), 213-232, Contemp. Math. 99, Amer. Math. Soc., Providence, RI, 1989.

Visual Computing and Vitual Reality Key Laboratory, Sichuan Normal University, Chengdu, 610066, China

Current address: College of Mathematics and Software Science, Sichuan Normal University, Chengdu, 610066, China

E-mail address: $\mathrm{zhu}_{-}$shihui2008@163.com

Visual Computing and Vitual Reality Key Laboratory, Sichuan Normal University, Chengdu, 610066, China

College of Mathematics, Southwest Jiaotong University, Chengdu, 610031, China 